\title{
Antimicrobial susceptibility monitoring of Mycoplasma hyopneumoniae isolated from seven European countries during 2015-2016
}

\author{
Anno de Jong a,b,*, Myriam Youala ${ }^{\text {a,c }}$, Ulrich Klein ${ }^{\text {a,d,1 }}$, Farid El Garch ${ }^{\text {a,e }}$, Hilde Moyaert ${ }^{\text {a,f }}$, \\ Shabbir Simjee $^{\mathrm{a}, \mathrm{d}}$, Dominiek Maes ${ }^{g}$, Miklós Gyuranecz ${ }^{\mathrm{h}}$, Andrew Pridmore ${ }^{\mathrm{i}}$, Jill R. Thomson ${ }^{\mathrm{j}}$, \\ Roger D. Ayling ${ }^{k}$, on behalf of the MycoPath Study Group \\ ${ }^{a}$ MycoPath Study Group, c/o CEESA, 168 Av de Tervueren, 1150 Brussels, Belgium \\ ${ }^{\mathrm{b}}$ Consultant to CEESA, Brussels, Belgium \\ ${ }^{\mathrm{c}}$ Virbac, Carros, France \\ d Elanco Animal Health, Basingstoke, UK \\ ${ }^{\mathrm{e}}$ Vétoquinol SA, Lure, France \\ ${ }^{\mathrm{f}}$ Zoetis, Zaventem, Belgium \\ ${ }^{\mathrm{g}}$ Faculty of Veterinary Medicine, Ghent University, Ghent, Belgium \\ ${ }^{\mathrm{h}}$ Institute for Veterinary Medical Research CAR, Budapest, Hungary \\ ${ }^{\mathrm{i}}$ Don Whitley Scientific, Bingley, UK \\ ${ }^{\mathrm{j}}$ Scottish Agriculture College (Consulting) Veterinary Services, Edinburgh, UK \\ ${ }^{\mathrm{k}}$ Mycoplasma Consultant, Sandhurst, Berkshire, UK
}

\section{A R T I C L E I N F O}

\section{Keywords:}

Antimicrobial susceptibility

Surveillance

Minimal inhibitory concentrations

Mycoplasma hyopneumoniae

Porcine enzootic pneumonia

\begin{abstract}
A B S T R A C T
Mycoplasma hyopneumoniae is the causative agent of porcine enzootic pneumonia, a chronic respiratory disease, causing significant economic losses. Results from the 2015-2016 MycoPath pan-European antimicrobial susceptibility monitoring survey of $M$. hyopneumoniae are presented. In total, 147 M. hyopneumoniae porcine isolates from Belgium, France, Germany, Great Britain, Hungary, Italy, and Spain were tested. One isolate per farm was retained from pigs that had not been recently treated with antimicrobial agents. The minimal inhibitory concentration (MIC) of 13 antimicrobial agents was determined in a central laboratory using a broth microdilution method, with Friis Medium, incubated at $35 \pm 1{ }^{\circ} \mathrm{C}$ for 5-12 days. M. hyopneumoniae NCTC 10110 was used as Quality Control. $\mathrm{MIC}_{50} / \mathrm{MIC}_{90}(\mathrm{mg} / \mathrm{L})$ values were: enrofloxacin 0.06/1; marbofloxacin 0.06/2; spiramycin 0.06/ 0.25 ; tulathromycin $\leq 0.001 / 0.004$; gamithromycin $0.06 / 0.5$; tylosin $0.016 / 0.06$; tilmicosin $0.06 / 0.5$; florfenicol $0.5 / 1$; doxycycline $0.25 / 1$; oxytetracycline $0.25 / 2$; lincomycin $0.06 / 0.25$; tiamulin $0.016 / 0.06$ and valnemulin $\leq 0.001 / 0.004$. Compared with the data from 2010 to 2012 MycoPath study (50 isolates), MIC $_{50 / 90}$ results were similar and the majority were within \pm two dilution steps, except for the $\mathrm{MIC}_{50}$ of oxytetracycline which is more than two dilution steps higher in the present study. Between-country comparisons show some differences in the MIC values for the fluoroquinolones, tulathromycin and tylosin, but the limited sample size per country precludes performing meaningful country comparisons for several countries. Standardized laboratory methods and interpretive criteria for MIC testing of veterinary mycoplasmas are clearly needed; there are currently no clinical breakpoints available to facilitate data interpretation and correlation of MICs with in vivo efficacy.
\end{abstract}

\section{Introduction}

Mycoplasma hyopneumoniae is the cause of enzootic pneumonia in pigs and one of the major pathogens involved in the porcine respiratory disease complex (PRDC). The pathogen causes major economic losses to the pig industry worldwide, mainly due to reduced performance, higher antimicrobial use and predisposing the animals to other respiratory infections (Thacker and Minion, 2012; Pieters and Maes, 2019), with bacteria including Pasteurella multocida, Actinobacillus pleuropneumoniae and Streptococcus suis (Maes et al., 2008; Marois et al., 2009;

\footnotetext{
* Corresponding author at: c/o CEESA, 168 Av de Tervueren, 1150 Brussels, Belgium.

E-mail address: anno.jong-bayer@kpnmail.nl (A. de Jong).

1 Present address of Ulrich Klein: Huvepharma, Antwerp, Belgium.
} 
Marois-Créhan et al., 2020). M. hyopneumoniae potentiates the severity of Betaarterivirus suid 1 (International Committee on Taxonomy of Viruses (ICTV, 2019), formerly named porcine reproductive and respiratory syndrome virus (PRRSV) (Thacker et al., 1999; Marois-Créhan et al., 2020), and porcine circovirus type 2 (PCV2) associated pneumonia in pigs (Seo et al., 2014; Marois-Créhan et al., 2020). Commercial vaccines are available but do not prevent colonisation of the respiratory tract (Maes et al., 2008; Arsenakis et al., 2017). Consequently antimicrobial agents are often needed to treat and help control infections (Maes et al., 2018). Various antimicrobial agents are effective and licensed for treating $M$. hyopneumoniae infections. Potentially active antimicrobial agents against $M$. hyopneumoniae include aminoglycosides/aminocyclitols, fluoroquinolones, lincosamides, macrolides, phenicols, pleuromutilins and tetracyclines (Gautier-Bouchardon, 2020; Maes et al., 2020). Mycoplasma species lack a cell wall and are therefore refractory to all antimicrobial agents that target the cell wall such as $\beta$-lactams (Wu et al., 1997; Lysnyansky and Ayling, 2016). Sulfonamide antimicrobial agents are also ineffective because mycoplasmas do not synthesize folic acid (Gautier-Bouchardon, 2018).

Antimicrobial resistance of bacterial pathogens is of concern for the antimicrobial therapy of both humans and animals. Harmonized and continuous monitoring of antimicrobial susceptibility trends over time is an important component of stewardship to ensure long-term antimicrobial efficacy. However, recent antimicrobial susceptibility data of M. hyopneumoniae is rather limited, in contrast to Mycoplasma bovis susceptibility data (e.g., Heuvelink et al., 2016; Cai et al., 2019). Moreover, there are limitations in the available M. hyopneumoniae data, as published studies either have a limited number of isolates, or are only from one country (Hannan et al., 1997a; Vicca et al., 2004; Tavío et al., 2014). In other studies the epidemiological sampling framework, or herd information is either limited or absent (Hannan et al., 1997a, 1997b). Historic M. hyopneumoniae antimicrobial susceptibility data may be of limited value today due to decades of altered antimicrobial consumption. In none of the European national, ongoing programmes such as GERM-Vet (Germany), RESAPATH (France) or UK-VARSS (Great Britain) are these organisms included. Hence, there is need for recent $M$. hyopneumoniae antimicrobial susceptibility data to resolve the above limitations.

The present study was conducted as part of the Centre Européen d`Etudes pour la Santé Animale (CEESA) monitoring programmes (de Jong et al., 2013). The MycoPath programme aims to create a pan-European collection of representative Mycoplasma pathogens isolated from clinical cases of diseased poultry (Mycoplasma gallisepticum and Mycoplasma synoviae), cattle ( $M$. bovis), and pigs (M. hyopneumoniae) that have not recently been exposed to antimicrobial agents. These isolates are used to monitor the in vitro susceptibility of licensed antimicrobial agents and can also be used by pharmaceutical companies to assess the in vitro effectiveness of any new antimicrobial agents being developed. Antimicrobial susceptibilities of $M$. hyopneumoniae recovered from seven European countries are presented here. This is the second study (MycoPath II) to include M. hyopneumoniae as part of the CEESA monitoring programmes, so comparisons can be made with the previous study (MycoPath I; Klein et al., 2017).

\section{Methodology}

\subsection{Mycoplasma hyopneumoniae collection}

During 2015 to 2016 M. hyopneumoniae isolates were obtained following post-mortem sampling of pigs. Pigs were aged from three weeks to seven months with clinical signs of respiratory disease or from slaughterhouse lung samples with pathology consistent with enzootic pneumonia. Samples had to meet specific criteria which included lack of antimicrobial treatment in the previous 15 days, with only one isolate per farm. The participating laboratories were from Belgium, France,
Germany, Great Britain, Hungary, Italy and Spain. In an attempt to achieve identical numbers of isolates from the participating countries, a target number of 40 isolates were indicated for each country. The majority of the participating countries followed their standard Mycoplasma culture isolation and molecular identification procedures (Mattisson et al., 1995; Moronato et al., 2017); the samples from Germany and Spain as well as some of the British samples were sent for isolation and identification to the central laboratory (Don Whitley Scientific, Bingley, UK). Isolates were stored at $-70 \pm 10^{\circ} \mathrm{C}$, before transfer to the central laboratory for antimicrobial susceptibility testing.

At the central laboratory the isolates were checked for viability with M. hyopneumoniae being cultured in Friis medium (Friis, 1975). Each culture was grown and the viable count determined by plating onto the agar medium so that the cell density could be adjusted to $10^{6} \mathrm{CFU} / \mathrm{mL}$ for the minimal inhibitory concentration (MIC) tests. In addition to the identification of the British, German and Spanish isolates at the central laboratory, identity was confirmed for eight randomly selected M. hyopneumoniae isolates (5.4\%) using a duplex PCR method that identified both $M$. hyopneumoniae and $M$. hyorhinis giving a $430 \mathrm{bp}$ and 346 bp amplicon, respectively (Barate et al., 2012). The identity of the eight isolates was confirmed.

\subsection{Antimicrobial testing}

Antimicrobial susceptibility testing used a broth microdilution method to determine the MICs to 13 antimicrobial agents. The antimicrobial agents included in this study belong to six frequently used chemical classes: the phenicols (florfenicol); the fluoroquinolones (enrofloxacin, marbofloxacin); the macrolides and modified macrolides (gamithromycin, spiramycin, tilmicosin, tulathromycin, tylosin); the pleuromutilins (tiamulin and valnemulin); the tetracyclines (doxycycline and oxytetracycline) and the lincosamides (lincomycin). The antimicrobial agents were obtained as: doxycycline hyclate; enrofloxacin; florfenicol; lincomycin hydrochloride; marbofloxacin; oxytetracycline hydrochloride; spiramycin; tiamulin fumarate; tilmicosin; tylosin tartrate (all from Sigma-Aldrich, UK); gamithromycin (Merial; France); tulathromycin (Pfizer, USA); and valnemulin hydrogen tartrate (European Directorate for the Quality of Medicines and Healthcare, France). The preparation of the stock solutions of the antimicrobial agents has been described recently (Klein et al., 2017, 2019), except for doxycycline, lincomycin and tilmicosin. Doxycycline and lincomycin were prepared in deionized water, whereas tilmicosin was dissolved in $95 \%$ ethanol before being made to the correct final volume with deionized water. It should be noted that the importance of correct buffering of tulathromycin in the MIC test to avoid artificial MIC shifts (Godinho, 2008) was respected in our study.

The determination of MIC values for each isolate has been performed as previously described (Klein et al., 2017). In short, the MICs of the 13 antimicrobial agents were determined using a broth microdilution method, with Friis Medium (without bacitracin and methicillin), incubated at $35 \pm 1{ }^{\circ} \mathrm{C}$. The incubation period required to produce clearly readable MIC endpoints varied between 5 and 12 days, according to the individual M. hyopneumoniae strains. The type strain $M$. hyopneumoniae NCTC 10110 (ATCC 25934) was used as a Quality Control (QC) for all MIC testing. In a number of cases MICs of the test isolates could not be assessed due to poor growth or loss of viability. In these cases the incomplete datasets were indicated in Tables 1 and 2 . In cases where the MIC results obtained for an antimicrobial agent against one or more strains of M. hyopneumoniae deviated markedly from the MICs obtained against the majority of strains, the MIC test was repeated twice. In such cases, the reported MIC value was obtained on at least two separate occasions. 
Table 1

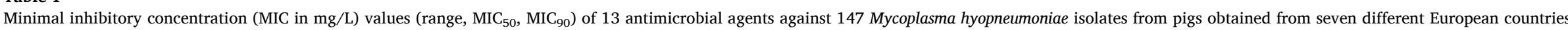
between 2015 and 2016.

\begin{tabular}{|c|c|c|c|c|c|c|c|c|c|c|c|c|c|c|}
\hline $\begin{array}{l}\text { Country of } \\
\text { origin }\end{array}$ & $\begin{array}{l}\text { MIC } \\
\text { parameter }\end{array}$ & ENR & MAR & DOX & отс & FFC & LC & TIA & VAL & GAM & TUL & SPM & TIL & TYL \\
\hline \multirow{4}{*}{$\begin{array}{l}\text { Belgium (40 } \\
\text { isolates) }\end{array}$} & Range & $0.008-2$ & $0.008-2$ & $0.008-1$ & $0.008-2$ & $0.002-1$ & $0.002->64$ & $0.004-0.25$ & $\leq 0.001-0.008$ & $\leq 0.001-1$ & $\leq 0.001-\geq 128$ & $0.002-8$ & $0.002-64$ & $0.002-16$ \\
\hline & $\mathrm{MIC}_{50}$ & 0.063 & 0.031 & 0.25 & 0.5 & 0.25 & 0.063 & 0.016 & $\leq 0.001$ & 0.063 & $\leq 0.001$ & 0.063 & 0.031 & 0.004 \\
\hline & $\mathrm{MIC}_{90}$ & 0.25 & 0.5 & 0.5 & 1 & 0.5 & 0.5 & 0.063 & 0.002 & 0.5 & 8 & 0.5 & 0.5 & 0.5 \\
\hline & $\begin{array}{l}\text { isolates } \\
\text { tested }\end{array}$ & 38 & 40 & 40 & 40 & 38 & 39 & 39 & 39 & 37 & 39 & 37 & 40 & 40 \\
\hline \multirow{4}{*}{$\begin{array}{c}\text { France }(10 \\
\text { isolates })\end{array}$} & Range & $0.008-0.25$ & $0.016-0.063$ & $0.031-0.5$ & $0.002-0.031$ & $0.125-0.5$ & $0.004-0.125$ & $0.008-0.031$ & All $\leq 0.001$ & $0.016-0.5$ & $\leq 0.001-0.002$ & $0.063-0.25$ & $0.125-0.5$ & $0.031-0.063$ \\
\hline & $\mathrm{MIC}_{50}$ & 0.031 & 0.031 & 0.125 & 0.002 & 0.25 & 0.063 & 0.016 & $\leq 0.001$ & 0.5 & 0.002 & 0.125 & 0.25 & 0.063 \\
\hline & $\mathrm{MIC}_{90}$ & 0.063 & 0.063 & 0.25 & 0.016 & 0.5 & 0.125 & 0.016 & $\leq 0.001$ & 0.5 & 0.002 & 0.25 & 0.5 & 0.063 \\
\hline & $\begin{array}{l}\text { isolates } \\
\text { tested }\end{array}$ & 10 & 10 & 10 & 10 & 10 & 10 & 10 & 10 & 10 & 10 & 10 & 10 & 10 \\
\hline \multirow{4}{*}{$\begin{array}{l}\text { Germany (15 } \\
\text { isolates) }\end{array}$} & Range & $0.016-2$ & $0.031-2$ & $0.031-1$ & $0.016-1$ & $0.031-1$ & $0.002-32$ & $0.004-0.125$ & $\leq 0.001-0.004$ & $0.002-2$ & $\leq 0.001-8$ & $0.004-16$ & $0.004-\geq 128$ & $0.002-16$ \\
\hline & $\mathrm{MIC}_{50}$ & 0.25 & 0.5 & 0.063 & 0.125 & 0.5 & 0.125 & 0.031 & $\leq 0.001$ & 0.031 & $\leq 0.001$ & 0.063 & 0.125 & 0.016 \\
\hline & $\mathrm{MIC}_{90}$ & 2 & 2 & 0.25 & 0.25 & 1 & 0.5 & 0.125 & 0.002 & 0.5 & 0.008 & 16 & 0.5 & 0.063 \\
\hline & $\begin{array}{l}\text { isolates } \\
\text { tested }\end{array}$ & 15 & 15 & 15 & 15 & 15 & 15 & 15 & 15 & 15 & 15 & 15 & 15 & 15 \\
\hline \multirow{4}{*}{$\begin{array}{r}\text { Great Britain } \\
\quad(25 \text { isolates })\end{array}$} & Range & $0.004-1$ & $0.004-1$ & $0.004-1$ & $0.031-4$ & $0.002-1$ & $0.002-\geq 128$ & $\leq 0.001-0.063$ & $\leq 0.001-0.004$ & $\begin{array}{l}\leq 0.001- \\
\geq 128\end{array}$ & $\leq 0.001-16$ & $0.008-4$ & $0.004-64$ & $\leq 0.001-32$ \\
\hline & $\mathrm{MIC}_{50}$ & 0.016 & 0.031 & 0.5 & 0.5 & 0.5 & 0.063 & 0.031 & $\leq 0.001$ & 0.125 & 0.002 & 0.125 & 0.125 & 0.031 \\
\hline & $\mathrm{MIC}_{90}$ & 0.125 & 0.5 & 1 & 1 & 1 & 0.5 & 0.063 & 0.002 & 1 & 0.008 & 4 & 1 & 0.125 \\
\hline & $\begin{array}{l}\text { isolates } \\
\text { tested }\end{array}$ & 25 & 25 & 25 & 25 & 25 & 25 & 25 & 25 & 25 & 25 & 25 & 25 & 25 \\
\hline \multirow{4}{*}{$\begin{array}{l}\text { Hungary (43 } \\
\text { isolates) }\end{array}$} & Range & $0.008-4$ & $0.004-4$ & $0.031-1$ & $0.063-4$ & $0.063-1$ & $0.002-\geq 128$ & $0.002-0.125$ & $\leq 0.001-0.004$ & $0.004-0.5$ & $\leq 0.001-0.002$ & $0.008-16$ & $0.004-64$ & $0.004-32$ \\
\hline & $\mathrm{MIC}_{50}$ & 0.125 & 0.5 & 0.25 & 0.5 & 0.5 & 0.125 & 0.016 & 0.002 & 0.031 & $<0.001$ & 0.063 & 0.063 & 0.031 \\
\hline & $\mathrm{MIC}_{90}$ & 2 & 2 & 1 & 2 & 1 & 0.25 & 0.063 & 0.004 & 0.125 & 0.002 & 0.25 & 0.25 & 0.063 \\
\hline & $\begin{array}{l}\text { isolates } \\
\text { tested }\end{array}$ & 43 & 43 & 43 & 43 & 43 & 43 & 43 & 43 & 43 & 43 & 43 & 43 & 43 \\
\hline \multirow{4}{*}{ Italy (5 isolates) } & Range & $1-2$ & $2-2$ & $0.25-1$ & $0.031-0.25$ & $0.5-0.5$ & $0.002-0.008$ & $0.016-0.063$ & All $\leq 0.001$ & $0.004-0.063$ & All $\leq 0.001$ & $0.004-0.031$ & $0.002-0.063$ & $0.002-0.016$ \\
\hline & $\mathrm{MIC}_{50}$ & 1 & 2 & 0.5 & 0.031 & 0.5 & 0.004 & 0.016 & $\leq 0.001$ & 0.004 & $\leq 0.001$ & 0.008 & 0.004 & 0.002 \\
\hline & $\mathrm{MIC}_{90}$ & ND & ND & ND & ND & ND & ND & ND & ND & ND & ND & ND & ND & ND \\
\hline & $\begin{array}{l}\text { isolates } \\
\text { tested }\end{array}$ & 2 & 2 & 5 & 5 & 5 & 5 & 5 & 5 & 2 & 4 & 5 & 4 & 5 \\
\hline \multirow{4}{*}{$\begin{array}{l}\text { Spain (9 } \\
\text { isolates) }\end{array}$} & Range & $0.031-2$ & $0.12-4$ & $0.12-1$ & $0.063-1$ & $0.004-0.5$ & $0.002-0.12$ & $0.002-0.12$ & $\leq 0.001-0.002$ & $0.004-2$ & $\leq 0.001-0.008$ & $0.008-0.5$ & $0.031-0.5$ & $0.016-16$ \\
\hline & $\mathrm{MIC}_{50}$ & 1 & 2 & 0.5 & 0.25 & 0.25 & 0.031 & 0.031 & $\leq 0.001$ & 0.25 & 0.002 & 0.125 & 0.25 & 0.031 \\
\hline & $\mathrm{MIC}_{90}$ & ND & ND & ND & ND & ND & ND & ND & ND & ND & ND & ND & ND & ND \\
\hline & $\begin{array}{l}\text { 1solates } \\
\text { tested }\end{array}$ & 9 & 9 & 9 & 9 & 9 & 9 & 9 & 9 & 9 & 9 & 9 & 9 & 9 \\
\hline \multirow{4}{*}{$\begin{array}{l}\text { All (147 } \\
\text { isolates) }\end{array}$} & Range & $0.004-4$ & $0.004-4$ & $0.004-1$ & $0.002-4$ & $0.002-1$ & $0.002-\geq 128$ & $\leq 0.001-0.25$ & $\leq 0.001-0.008$ & $\begin{array}{l}<0.001- \\
\geq 128\end{array}$ & $\leq 0.001-\geq 128$ & $0.002-16$ & $0.002-\geq 128$ & $\leq 0.001-32$ \\
\hline & $\mathrm{MIC}_{50}$ & 0.063 & 0.063 & 0.25 & 0.25 & 0.5 & 0.063 & 0.016 & $\leq 0.001$ & 0.063 & $\leq 0.001$ & 0.063 & 0.063 & 0.016 \\
\hline & $\mathrm{MIC}_{90}$ & 1 & 2 & 1 & 2 & 1 & 0.25 & 0.063 & 0.004 & 0.5 & 0.004 & 0.25 & 0.5 & 0.063 \\
\hline & $\begin{array}{l}\text { isolates } \\
\text { tested }\end{array}$ & 142 & 144 & 147 & 147 & 145 & 146 & 146 & 146 & 141 & 145 & 144 & 146 & 147 \\
\hline QC & MIC range & $\begin{array}{l}0.031-0.063 \\
(12)\end{array}$ & $0.063(7)$ & $\begin{array}{l}0.125-0.5 \\
(9)\end{array}$ & $0.25-1(8)$ & $\begin{array}{l}0.5-1 \\
(10)\end{array}$ & $\begin{array}{l}0.25-0.5 \\
(10)\end{array}$ & $\begin{array}{l}0.016-0.125 \\
(12)\end{array}$ & $\begin{array}{l}\leq 0.001-0.004 \\
(10)\end{array}$ & $\begin{array}{l}0.063-0.5 \\
(12)\end{array}$ & $\begin{array}{l}0.002-0.008 \\
(11)\end{array}$ & $\begin{array}{l}0.031-0.25 \\
(13)\end{array}$ & $\begin{array}{l}0.063-0.25 \\
(12)\end{array}$ & $\begin{array}{l}0.031-0.125 \\
(14)\end{array}$ \\
\hline
\end{tabular}

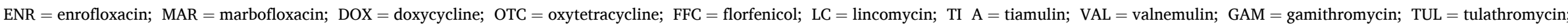
$\mathrm{SPM}=$ spiramycin $; \mathrm{TIL}=$ tilmicosin $; \mathrm{TYL}=$ tylosin.

$\mathrm{ND}=\mathrm{MIC}_{90}$ not determined. QC: Mycoplasma hyopneumoniae (NCTC 10,110) was used as a quality control standard in this study; number of MIC determinations is indicated in parentheses. 


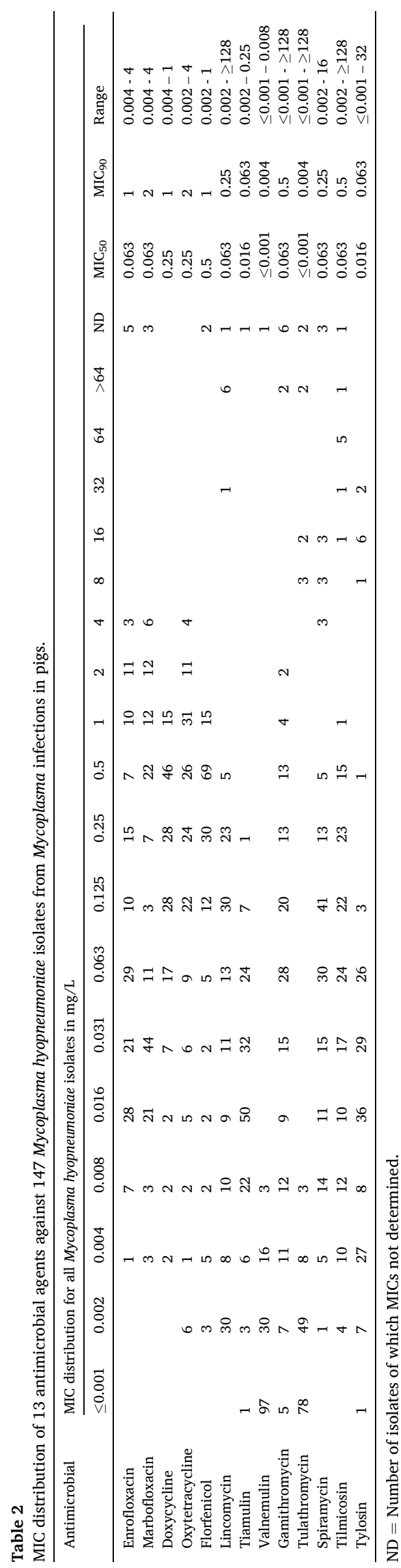

\section{Results}

\subsection{Mycoplasma hyopneumoniae collection}

Despite intensive efforts by participants in each country, it was not possible for all of them to obtain the target number $(n=40)$ of isolates, mainly because animals were treated prior to sampling. Records of all samples indicated that $85.9 \%$ of the sampled pigs had not been exposed to antibacterial treatment for at least 15 days prior to sampling. The remaining $14.1 \%$ of the samples were from animals with a treatment status characterized as "unknown". Ultimately we succeeded to obtain a total of $147 \mathrm{M}$. hyopneumoniae isolates associated with respiratory disease, 40 from Belgium, 10 from France, 15 from Germany, 25 from Great Britain, 43 from Hungary, 5 from Italy and 9 from Spain. Sixty-one isolates (41.5\%) were recovered from samples taken in 2015; 86 isolates (58.5 \%) were from samples of 2016. The very limited number of isolates for Italy and Spain prevents comparison with other countries. Therefore comparisons in this results section are limited to countries with ten or more isolates. The majority of the lung samples $(72.8 \%)$ were taken at abattoirs, from tissue lesions of fatteners at an age of $25-28$ weeks old. The herd size varied widely, e.g., in Belgium from 80 to 2400 pigs, in Germany from 520 to 18,000 pigs and in Hungary from 120 to 13,500 pigs. Each isolate included in the study was from a different farm.

\subsection{Antimicrobial susceptibilities (Tables 1 and 2)}

For each antimicrobial agent, $M$. hyopneumoniae MIC results for individual countries and for all isolates are presented as MIC range, $\mathrm{MIC}_{50}$ and MIC $_{90}$ in Table 1 . The number of isolates tested, which varies per country per antimicrobial due to a few non-viable isolates, is also listed. Table 1 also includes the MIC values of the reference type strain. Frequency distributions of MIC results are presented in Table 2.

For both of the fluoroquinolones enrofloxacin (142 isolates) and marbofloxacin (144 isolates) a bimodal or multimodal MIC distribution was observed and the $\mathrm{MIC}_{50}$ for these isolates was $0.063 \mathrm{mg} / \mathrm{L}$, with an $\mathrm{MIC}_{90}$ value of 1 and $2 \mathrm{mg} / \mathrm{L}$ respectively. Belgium, France and Great Britain had $\mathrm{MIC}_{50}$ and $\mathrm{MIC}_{90}$ fluoroquinolone values that were $2-3 \mathrm{di}$ lutions lower than those for Germany and Hungary.

Doxycycline and oxytetracycline antimicrobial agents gave similar $\mathrm{MIC}_{50}$ and $\mathrm{MIC}_{90}$ values of $0.25 / 1$ and $0.25 / 2 \mathrm{mg} / \mathrm{L}$, respectively. However doxycycline MIC $_{50}$ and MIC $_{90}$ values for Germany were 0.063 and $0.25 \mathrm{mg} / \mathrm{L}$ whereas oxytetracyline MICs were lower for France at 0.002 and $0.016 \mathrm{mg} / \mathrm{L}$ respectively. Florfenicol had similar MIC range $0.002-1 \mathrm{mg} / \mathrm{L}$ and $\mathrm{MIC}_{50}$ values of 0.25 to $0.5 \mathrm{mg} / \mathrm{L}$, and $\mathrm{MIC}_{90}$ values of $0.5-1 \mathrm{mg} / \mathrm{L}$ for all countries. For all three compounds, a broad monomodal MIC distribution pattern was seen.

Belgium, Germany, Great Britain and Hungary had some isolates with high lincomycin MIC values at 32 and $\geq 128 \mathrm{mg} / \mathrm{L}$, although the $\mathrm{MIC}_{50}$ and $\mathrm{MIC}_{90}$ values all had a range of 0.063 to $0.125 \mathrm{mg} / \mathrm{L}$ and 0.125 to $0.5 \mathrm{mg} / \mathrm{L}$, respectively. Low MIC ranges and $\mathrm{MIC}_{50 / 90}$ values were observed for both pleuromutilin antimicrobial agents from all countries: MIC range of $\leq 0.001$ to $0.25 \mathrm{mg} / \mathrm{L}$ and $\mathrm{MIC}_{50 / 90} 0.016$ / $0.063 \mathrm{mg} / \mathrm{L}$ for tiamulin and MIC range $\leq 0.001$ to $0.008 \mathrm{mg} / \mathrm{L}$ and $\mathrm{MIC}_{50 / 90} \leq 0.001 / 0.004 \mathrm{mg} / \mathrm{L}$ for valnemulin. Overall, a multimodal MIC pattern was observed.

The macrolides and modified macrolides displayed $\mathrm{MIC}_{50 / 90}$ values of $0.063 / 0.5 \mathrm{mg} / \mathrm{L}$ for gamithromycin; $0.063 / 0.25 \mathrm{mg} / \mathrm{L}$ for spiramycin; $0.063 / 0.5 \mathrm{mg} / \mathrm{L}$ for tilmicosin; $\leq 0.001 / 0.004 \mathrm{mg} / \mathrm{L}$ for tulathromycin and $0.016 / 0.063 \mathrm{mg} / \mathrm{L}$ for tylosin. For gamithromycin France had the highest MIC $_{50}$ value at $0.5 \mathrm{mg} / \mathrm{L}$ but the $\mathrm{MIC}_{90}$ value at $0.5 \mathrm{mg} / \mathrm{L}$ was comparable to the other countries with Great Britain having the highest $\mathrm{MIC}_{90}$ value of $1 \mathrm{mg} / \mathrm{L}$. Great Britain also had the highest $\mathrm{MIC}_{90}$ value of $1 \mathrm{mg} / \mathrm{L}$ for tilmicosin compared to $0.25-0.5 \mathrm{mg} /$ $\mathrm{L}$ for the other countries. For tulathromycin, Belgium had a $\mathrm{MIC}_{90}$ value of $8 \mathrm{mg} / \mathrm{L}$ compared to $0.002-0.008 \mathrm{mg} / \mathrm{L}$ for other countries. 
Multimodal MIC patterns were observed.

\section{Discussion}

In this study, 13 antimicrobial agents were tested against 147 M. hyopneumoniae isolates from seven European countries, and was therefore more extensive than the previous study (Klein et al., 2017) in which 9 antimicrobial agents were tested against 50 isolates from three countries. To ensure consistency, a central laboratory performed all the MIC testing and used the same method as described previously (Klein et al., 2017), following the guidelines of Hannan (2000). This approach facilitates broad comparisons between the two studies. Although a few isolates gave high MIC values, overall the $\mathrm{MIC}_{50}$ and $\mathrm{MIC}_{90}$ values for all of the antimicrobial agents are low, less than 0.5 and $2 \mathrm{mg} / \mathrm{L}$ respectively when compared to MICs obtained for M. bovis (Ayling et al., 2014; Klein et al., 2017; 2019). The authors are unaware of any collection of M. hyopneumoniae isolates that is as representative of the European population of pigs, both in size and geographic diversity.

MIC interpretation criteria are not available for M. hyopneumoniae. Generally antimicrobial susceptibility testing for veterinary Mycoplasma species lacks quality control strains with specified MIC ranges, test methods and breakpoints (Maes et al., 2018), although guidelines have been published (Hannan, 2000). Standards for the Mycoplasma testing of significant clinical infections in humans (Mycoplasma pneumoniae, Mycoplasma hominis, and Ureaplasma urealyticum) have been published (CLSI, 2011; Waites et al., 2012). However, the growth requirements of these Mycoplasma species that occur in humans differ significantly from $M$. hyopneumoniae. Therefore the growth media that are suitable for testing these human derived Mycoplasma species cannot be applied to livestock species. It is important to note that, in addition to the CLSI standards for human mycoplasmas, a EUCAST Veterinary Subcommittee on Antimicrobial Susceptibility Testing (VetCAST) has recently been established. One of the remits of VetCAST is to initiate and coordinate EU research aimed at filling the current gaps on veterinary-specific breakpoints including epidemiological cut-offs (ECOFFs) values (Toutain et al., 2017).

It should be underlined that the numbers of isolates tested are too small to draw definitive conclusions about potential country differences. In addition, all QC MIC values were within two dilutions, except for gamithromycin, spiramycin and tiamulin (three dilutions). The MIC ranges for these three compounds obtained for the type culture strain precludes valid data about subtle MIC shifts. The central laboratory standardised the testing so these differences are hard to explain. It might be due to the low number of QC tests (7-14). Hannan (2000) reported variations in MIC values from 0.01 to $0.1 \mathrm{mg} / \mathrm{L}$ for tiamulin and $0.25-2.5 \mathrm{mg} / \mathrm{L}$ for spiramycin due to differences in inoculum size, other factors such as growth phase and incubation times could also affect the MIC value. However, the reproducibility of the MICs harvested from the field isolates was high. Further work is required to standardize the MIC test methodology for veterinary Mycoplasma species.

With respect to several potential MIC differences between countries (Table 1), it is tempting to investigate the relationship between antimicrobial consumption for the treatment of bacterial infections and the MIC levels. Regrettably, historic data on the antimicrobial consumption on the specific farms where the samples were obtained is not available. Several years ago the European Surveillance of Veterinary Antimicrobial Consumption (ESVAC) was established to collect data on the use of antimicrobial agents in animals in Europe (EMA, 2019). The uniform record of the national antibiotic consumption in veterinary medicine is highly valuable. However, only the total consumption per country per annum is being reported. The lack of consumption data per animal species or clinical indication such as mycoplasmoses in pigs, limits attempts to explain any differences in antimicrobial susceptibilities between countries and over time. In the meantime quantification of antibiotic use has become mandatory in several countries, e.g. Belgium, The Netherlands and Denmark. This will facilitate future investigations into the association between antibiotic use and antimicrobial susceptibility results obtained for $M$. hyopneumoniae isolates.

Comparison of the $\mathrm{MIC}_{50}$ and $\mathrm{MIC}_{90}$ values between this study (MycoPath II) and that of Klein et al. (2017) (MycoPath I) showed minor differences. When individual country data is examined (Table 1), there is an apparent increase in the maximum MIC of valnemulin from 0.002 to $0.004 \mathrm{mg} / \mathrm{L}$ for Great Britain and to $0.008 \mathrm{mg} / \mathrm{L}$ for Belgium. For tulathromycin the overall $\mathrm{MIC}_{50}$ and $\mathrm{MIC}_{90}$ remain low at $\leq 0.001$ and $0.004 \mathrm{mg} / \mathrm{L}$ respectively, but some isolates from Belgium and Great Britain had tulathromycin MIC values of $16 \mathrm{mg} / \mathrm{L}$ and as high as $\geq 128 \mathrm{mg} / \mathrm{L}$. Although there are limitations and a risk of over interpretating data from this study, an increase in oxytetracycline $\mathrm{MIC}_{50}$ values for the three countries included in the previous studies from 0.063 to $0.5 \mathrm{mg} / \mathrm{L}$ for Belgium, 0.063 to $0.25 \mathrm{mg} / \mathrm{L}$ for Spain and 0.031 to $0.5 \mathrm{mg} / \mathrm{L}$ for Great Britain indicates a genuine rise in MIC levels.

Some MIC increases can be explained by the inclusion of additional countries in this study; for example Germany, Hungary and Italy had higher $\mathrm{MIC}_{50}$ and $\mathrm{MIC}_{90}$ values than Belgium, France and Great Britain to the fluoroquinolones enrofloxacin and marbofloxacin. In order to avoid any bias, we have therefore compared the MIC values of isolates from the three countries (Belgium, Great Britain and Spain) which were common in both the MycoPath I and MycoPath II surveys (Table 3). Compared to the 2010-2012 study, an $\mathrm{MIC}_{50}$ increase of three doubling dilutions is observed for oxytetracycline. MIC 90 values increases of two doubling dilutions were noted only for oxytetracycline, valnemulin and tylosin as compared to the 2010-2012 study. However, these minor shifts should not be over interpreted; most changes are within the normal fluctuation indicated by CLSI of \pm one dilution step.

The results of the NCTC strain 10110 used for QC showed some differences between MycoPath I (Klein et al., 2017) and this study (data included in Table 1), particularly for oxytetracycline, tiamulin, tulathromycin and tylosin, where there appeared to be a general increase. For example, for oxytetracycline the MIC range was $0.125-0.25 \mathrm{mg} / \mathrm{L}$ in MycoPath I and 0.25-1 mg/L in MycoPath II. These small differences are hard to explain. It might be due to the limited number of QC tests in MycoPath I $(<5)$. One could also postulate that minor variations between reference strain batches between MycoPath I and MycoPath II could have resulted in variation in metabolism and growth rates affecting some antimicrobial agents differently, although QC was carried out on the commercially obtained media which supported $M$. hyopneumoniae growth. However, the reproducibility of the field isolates MIC values was high.

Another study (Felde et al., 2018) mainly tested isolates from Hungary $(n=40)$, Slovakia $(n=3)$ and Czech Republic $(n=1)$, using a similar broth microdilution method, but with Mycoplasma Experience broth medium (Mycoplasma Experience Ltd., Bletchingley, UK). With

\section{Table 3}

Comparison of Minimal Inhibitory Concentration (MIC in $\mathrm{mg} / \mathrm{L}$ ) data for $\mathrm{My}$ coplasma hyopneumoniae of three countries (Belgium, Great Britain and Spain) between this study (MycoPath II) and the previous study (MycoPath I; Klein et al., 2017).

\begin{tabular}{|c|c|c|c|c|c|c|}
\hline \multirow{2}{*}{ Antimicrobial agent } & \multicolumn{2}{|c|}{ MycoPath I } & \multirow[b]{2}{*}{$\mathrm{n}$} & \multicolumn{3}{|c|}{ MycoPath II } \\
\hline & $\mathrm{MIC}_{50}$ & MIC $_{90}$ & & $\mathrm{MIC}_{50}$ & $\mathrm{MIC}_{90}$ & $\mathrm{n}^{\mathrm{a}}$ \\
\hline Enrofloxacin & 0.031 & 0.5 & 50 & 0.063 & 1 & 72 \\
\hline Marbofloxacin & 0.031 & 0.5 & 50 & 0.031 & 1 & 74 \\
\hline Oxytetracycline & 0.063 & 0.25 & 50 & 0.5 & 1 & 74 \\
\hline Florfenicol & 0.25 & 0.5 & 50 & 0.25 & 0.5 & 72 \\
\hline Tiamulin & 0.016 & 0.063 & 50 & 0.016 & 0.063 & 73 \\
\hline Valnemulin & $\leq 0.001$ & $\leq 0.001$ & 50 & $\leq 0.001$ & 0.002 & 73 \\
\hline Tulathromycin & $\leq 0.001$ & $0.004^{\mathrm{b}}$ & 50 & $\leq 0.001$ & 0.008 & 73 \\
\hline Spiramycin & 0.063 & 0.25 & 50 & 0.063 & 0.5 & 71 \\
\hline Tylosin & 0.031 & 0.125 & 50 & 0.016 & 0.5 & 74 \\
\hline
\end{tabular}

a Number of Belgian isolates tested varied between 37 and 40, as indicated in Table 1.

b MIC $_{90}$ value recalculated from Klein et al. (2017). 
the exception of valnemulin and enrofloxacin all of the antimicrobial agents tested by Felde et al. (2018) had higher MIC $_{50}$ and MIC 90 values. In comparison with the present study, the main difference between the tests was the growth medium. This may have influenced the test results, arguably the growth medium used may have given better control growth, however the medium formula is not published and it is possible that the medium contained anti-fungal and antimicrobial agents that may interfere with the MIC test. With longer incubation times the antimicrobial agents under test may start breaking down, or induce or select for antimicrobial resistance (Gautier-Bouchardon, 2018). However, the incubation time in the study of Felde et al. ranged from 4 to 14 days (Felde et al., 2018), whereas in our work it was 5-12 days. It is therefore unlikely that measuring any mycoplasmacidal effect rather than the inhibitory effect explains the differences. The MICs are performed on cells that should be in a planktonic state, as they are tested in a broth medium. However it is known that some M. hyopneumoniae strains have the ability to form biofilms, in which state they can potentially survive in ten-fold higher concentrations of antibiotics (Tassew et al., 2017). Although it is questionable whether M. hyopneumoniae will have enough time to form biofilms in MIC plates, this may be another possible explanation for some differences in results between published studies.

The occurrence of isolates with high MICs for lincomycin, gamithromycin, tulathromycin, tilmicosin and tylosin (MICs $\geq 32 \mathrm{mg} / \mathrm{L}$ ) might indicate that these isolates might have a resistance determinant. However, since breakpoints have not yet been determined for $M$. hyopneumoniae, the efficacy of an antimicrobial therapy cannot be predicted. It should be noticed that the MIC distribution only reflects the potency of an agent against a specific pathogen in vitro. The potential for developing antimicrobial resistance is evident from other Mycoplasma species, but also for $M$. hyopneumoniae which has been demonstrated in Tibet with macrolide resistance shown by mutations in the 23 rRNA gene (Qiu et al., 2018). Other reports have previously indicated antimicrobial resistance to some macrolides and lincosamides (Stakenborg et al., 2005; Thongkamkoon et al., 2013; Felde et al., 2018; Gautier-Bouchardon, 2018) and fluoroquinolones (Vicca et al., 2007; Felde et al., 2018). Mutations in the parC and gyrA genes have shown correlation with decreased fluoroquinolone susceptibility of M. hyopneumoniae. In other studies (on M. bovis isolates) alterations related to elevated tetracycline MICs were described in several positions of genes encoding $16 \mathrm{~S}$ rRNA and mutation in the rrs1 gene was related to a high spectinomycin MIC (Sulyok et al., 2017). Genetic mutations are known to relate to antimicrobial resistance and they demonstrated that the number of mutations also related directly to MIC values (Lerner et al., 2014). Mechanisms of antimicrobial resistance are outside the scope of this study. Studies need to be done to establish the relationship between genetic mutations and MIC values for M. hyopneumoniae and the potential role of plasmids, efflux or novel mechanisms for development of antimicrobial resistance by Mycoplasma species. Reviews of mechanisms of resistance and trends in antimicrobial susceptibility of Mycoplasma species were published by Lysnyansky and Ayling (2016) and Gautier-Bouchardon (2018).

It is important to monitor if isolates with high MIC values occur among $M$. hyopneumoniae and to determine the underlying resistance mechanism, so that only effective antimicrobial agents are used for therapy thus ensuring minimal use of antimicrobial agents by using targeted and correct treatments. In vitro MIC results do not necessarily correlate to the effectiveness of the antimicrobial agents in vivo and hence interpretation of the MIC distributions is made difficult as veterinary Mycoplasma species do not have defined clinical breakpoints. As seen when comparing the results from this study and that of Felde et al. (2018), even minor differences in methods may affect results, so this reinforces the urgent need for veterinary Mycoplasma-specific laboratory standards and clinical breakpoints for MIC data interpretation of $M y$ coplasma species. Comparison of the data from this study and the previous study indicates that most MIC values were similar; there was only a minor increase in MIC values for oxytetracycline (Klein et al., 2017). It however remains essential that the MycoPath monitoring programmes continue to monitor antimicrobial in vitro effectiveness.

\section{Funding}

The MycoPath project is a pan-European programme dedicated to the collection and to the monitoring of antimicrobial susceptibility of veterinary mycoplasmas from diseased food-producing animals. MycoPath is an initiative of, and is coordinated by the Executive Animal Health Study Center (CEESA) in Brussels. CEESA's membership is composed of international pharmaceutical companies researching and producing veterinary medicinal products. This study was funded by Bayer Animal Health GmbH (Germany), Boehringer Ingelheim (Germany), CEVA Santé Animale (France), Elanco Animal Health (UK), Merial (France), MSD Animal Health Innovation (Germany), Vetoquinol S.A. (France), Virbac (France) and Zoetis (Belgium). The authors thank the national co-ordinators and the national microbiological laboratories involved in the sampling and isolation procedures.

\section{Declaration of Competing Interest}

The authors report no declarations of interest.

\section{References}

Arsenakis, I., Michiels, A., Del Pozo Sacristan, R., Boyen, F., Haesebrouck, F., Maes, D., 2017. Mycoplasma hyopneumoniae vaccination at or shortly before weaning under field conditions: a randomised efficacy trial. Vet. Rec. 181, 19.

Ayling, R.D., Rosales, R.S., Barden, G., Gosney, F.L., 2014. Changes in antimicrobial susceptibility of Mycoplasma bovis isolates from Great Britain. Vet. Rec. 175, 486.

Barate, A.K., Lee, H.-Y., Jeong, H.-W., Truong, L.Q., Joo, H.-G., Hahn, T.-W., 2012. An improved multiplex PCR for diagnosis and differentiation of Mycoplasma hyopneumoniae and Mycoplasma hyorhinis. Korean J. Vet. Res. 52, 39-43.

Cai, H.Y., McDowall, R., Parker, L., Kaufman, E., Caswell, J.L., 2019. Changes in antimicrobial susceptibility profiles of Mycoplasma bovis over time. Can. J. Vet. Res. $83,34-41$.

Clinical and Laboratory Standards Institute, 2011. Methods for antimicrobial susceptibility testing for human mycoplasmas. Approved Guidelines 31 (19). CLSI document M43-A.

de Jong, A., Thomas, V., Klein, U., Marion, H., Moyaert, H., Simjee, S., Vallé, M., 2013. Pan-European resistance monitoring programmes encompassing food-borne bacteria and target pathogens of food-producing and companion animals. Int. J. Antimicrob. Agents 41, 403-409.

European Medicines Agency, European Surveillance of Veterinary Antimicrobial Consumption (ESVAC), 2019. Sales of Veterinary Antimicrobial Agents in 31 European Countries in 2017. Trends From 2010 to 2017. Ninth ESVAC Report. EMA/ $294674 / 2019$.

Felde, O., Kreizinger, Z., Sulyok, K.M., Hrivnák, V., Kiss, K., Jerzsele, A., Biksi, I., Gyuranecz, M., 2018. Antibiotic susceptibility testing of Mycoplasma hyopneumoniae field isolates from Central Europe for fifteen antibiotics by microbroth dilution method. PLoS One 13 e0209030.

Friis, N.F., 1975. Some recommendations concerning primary isolation of Mycoplasma suipneumoniae and Mycoplasma flocculare a survey. Nord. Vet. 27, 337-339.

Gautier-Bouchardon, A.V., 2018. Antimicrobial resistance in Mycoplasmaspp. Microbiol. Spectr. 6, 2018. ARBA-0030-2018.

Gautier-Bouchardon, A.V., 2020. Chapter 10: antimicrobial treatment of Mycoplasma hyopneumoniae infections. In: Maes, Dominiek, Sibila, Marina, Pieters, Maria (Eds.), Mycoplasmas in Swine. Acco Press Belgium, pp. 181-205.

Godinho, K.S., 2008. Susceptibility testing of tulathromycin: interpretative breakpoints and susceptibility of field isolates. Vet. Microbiol. 129, 426-432.

Hannan, P.C., 2000. Guidelines and recommendations for antimicrobial minimum inhibitory concentration (MIC) testing against veterinary mycoplasma species. Vet. Res. 31, 373-395.

Hannan, P.C.T., Windsor, H.M., Ripley, P.H., 1997a. In vitro susceptibilities of recent field isolates of Mycoplasma hyopneumoniae and Mycoplasma hyosynoviae to valnemulin (EconorR), tiamulin and enrofloxacin and the in vitro development of resistance to certain antimicrobials in Mycoplasma hyopneumoniae. Res. Vet. Sci. 63, 157-160.

Hannan, P.C.T., Windsor, G.D., de Jong, A., Schmeer, N., Stegemann, M., 1997b. Comparative susceptibilities of various animal-pathogenic mycoplasmas to fluoroquinolones. Antimicrob. Agents Chemother. 41, 2037-2040.

Heuvelink, A., Reugebrink, C., Mars, J., 2016. Antimicrobial susceptibility of Mycoplasma bovis isolates from veal calves and dairy cattle in the Netherlands. Vet. Microbiol. $189,1-7$.

International Committee on Taxonomy of Viruses (ICTV), 2019. Nidovirales Arteriviridae Porartevirus Porcine reproductive and respiratory syndrome virus 1 Nidovirales 
Arnidovirineae Arteriviridae Variarterivirinae Betaarterivirus Eurpobartevirus Betaarterivirus suid 1 PRRSV-1 rename and move species.

Klein, U., de Jong, A., Moyaert, H., El Garch, F., Leon, R., Richard-Mazet, A., Rose, M., Maes, D., Pridmore, A., Thomson, J.R., Ayling, R.D., 2017. Antimicrobial susceptibility monitoring of Mycoplasma hyopneumoniae and Mycoplasma bovis isolated in Europe. Vet. Microbiol. 204, 188-193. Erratum in: Vet. Microbiol. 2017, 208, 173.

Klein, U., de Jong, A., Youala, M., El Garch, F., Stevenin, C., Moyaert, H., Rose, M., Catania, S., Gyuranecz, M., Pridmore, A., Ayling, R.D., 2019. New antimicrobial susceptibility data from monitoring of Mycoplasma bovis isolated in Europe. Vet. Microbiol. 238, 108432.

Lerner, U., Amram, E., Ayling, R.D., Mikula, I., Gerchman, I., Harrus, S., Teff, D., Yogev, D., Lysnyansky, I., 2014. Acquired resistance to the 16-membered macrolides tylosin and tilmicosin by Mycoplasma bovis. Vet. Microbiol. 168, 365-371. Erratum in: Vet. Microbiol. 2015, 181, 335.

Lysnyansky, I., Ayling, R.D., 2016. Mycoplasma bovis: mechanisms of resistance and trends in antimicrobial susceptibility. Front. Microbiol. 27, 595 eCollection.

Maes, D., Segales, J., Meyns, T., Sibila, M., Pieters, M., Haesebrouck, F., 2008. Control of Mycoplasma hyopneumoniae infections in pigs. Vet. Microbiol. 126, 297-309.

Maes, D., Sibila, M., Kuhnert, P., Segalés, J., Haesebrouck, F., Pieters, M., 2018. Update on Mycoplasma hyopneumoniae infections in pigs: knowledge gaps for improved disease control. Transbound. Emerg. Dis. 65 (Suppl. 1), 110-124.

Maes, D., Boyen, F.J., Haesebrouck, F., Gautier-Bouchardon, A.V., 2020. Antimicrobial treatment of Mycoplasma hyopneumoniae infections. Vet. J. 259, 105474.

Marois, C., Gottschalk, M., Morvan, H., Fablet, C., Madec, F., Kobisch, M., 2009. Experimental infection of SPF pigs with Actinobacillus pleuropneumoniae serotype 9 alone or in association with Mycoplasma hyopneumoniae. Vet. Microbiol. 135, 283-291.

Marois-Créhan, C., Segalés, J., Holtkamp, D., Chae, C., Deblanc, C., Opriessnig, T., Fablet, C., 2020. Chapter 7: interactions of Mycoplasma hyopneumoniae with other pathogens and economic impact. In: Maes, Dominiek, Sibila, Marina, Pieters, Maria (Eds.), Mycoplasmas in Swine. Acco Press Belgium. In press.

Mattisson, J.G., Bergstrom, K., Wallgren, P., Johansson, K.E., 1995. Detection of Mycoplasma hyopneumoniae in nose swabs from pigs by in vitro amplification of the 16S rRNA gene. J. Clin. Microbiol. 33, 893-897.

Moronato, M.L., Ustulin, M., Vio, D., Nicholas, R.A.J., Catania, S., 2017. Diagnosis and control of a severe outbreak of lameness caused by Mycoplasma hyosynoviae in a closed pig unit. Vet. Rec. 0 e000500.

Pieters, M., Maes, D., 2019. Mycoplasmosis. In: Zimmerman, J.J., Karriker, L.A., Raminez, A., Schwartz, K.J., Stevenson, G.W., Zhang, J. (Eds.), Diseases of Swine. Eleventh Edition. Wiley Blackwell, pp. 863-883.

Qiu, G., Rui, Y., Zhang, J., Zhang, L., Huang, S., Wu, Q., Li, K., Han, Z., Liu, S., Li, J., 2018. Macrolide-resistance selection in Tibetan pigs with a high load of Mycoplasma hyopneumoniae. Microb. Drug Resist. 24, 1043-1049.
Seo, H.W., Park, S.J., Park, C., Chae, C., 2014. Interaction of porcine circovirus type 2 and Mycoplasma hyopneumoniae vaccines on dually infected pigs. Vaccine 32, 2480-2486.

Stakenborg, T., Vicca, J., Butaye, P., Maes, D., Minion, C., Peeters, J., de Kruif, A., Haesebrouck, F., 2005. Characterization of in vivo acquired resistance of Mycoplasma hyopneumoniae to macrolides and lincosamides. Microb. Drug Resist. 11, 290-294.

Sulyok, K.M., Kreizinger, Z., Wehmann, E., Lysnyansky, I., Banyai, K., Marton, S., Jerzsele, A., Ronai, Z., Turcsányi, I., Makrai, L., Jánosi, S., Nagy, S.A., Gyuranecz, M., 2017. Mutations associated with decreased susceptibility to seven antimicrobial families in field and laboratory-derived Mycoplasma bovis strains. Antimicrob. Agents Chemother. 61, e01983-16.

Tassew, D.D., Mechesso, A.F., Park, N.-H., Song, J.-B., Shur, J.-W., Park, S.-C., 2017. Biofilm formation and determination of minimum biofilm eradication concentration of antibiotics in Mycoplasma hyopneumoniae. J. Vet. Med. Sci. 79, 1716-1720.

Tavío, M.M., Poveda, C., Assunção, P., Ramírez, A.S., Poveda, J.B., 2014. In vitro activity of tylvalosin against Spanish field strains of Mycoplasma hyopneumoniae. Vet. Rec. $175,539$.

Thacker, E., Minion, C.F., 2012. Mycoplasmosis. In: Zimmerman, J.J., Karriker, L.A., Raminez, A., Schwartz, K.J., Stevenson, G.W. (Eds.), Diseases of Swine, 10th edn. Wiley-Blackwell Publishing, pp. 2850-2923.

Thacker, E., Halbur, P., Ross, R., Thanawongnuwech, R., Thacker, B., 1999. Mycoplasma hyopneumoniae potentiation of porcine reproductive and respiratory syndrome virusinduced pneumonia. J. Clin. Microbiol. 37, 620-627.

Thongkamkoon, P., Narongsak, W., Kobayashi, H., Pathanasophon, P., Kishima, M., Yamamoto, K., 2013. In vitro susceptibility of Mycoplasma hyopneumoniae field isolates and occurrence of fluoroquinolone, macrolides and lincomycin resistance. J. Vet. Med. Sci. 75, 1067-1070.

Toutain, P.-L., Bousquet-Mélou, A., Damborg, P., Ferran, A.A., Mevius, D., Pelligand, L., Veldman, K.T., Lees, P., 2017. En route towards European clinical breakpoints for veterinary antimicrobial susceptibility testing: a position paper explaining the VetCAST approach. Front. Microbiol. 8, 2344.

Vicca, J., Stakenborg, T., Maes, D., Butaye, P., Peeters, J., de Kruif, A., Haesebrouck, F., 2004. In vitro susceptibilities of Mycoplasma hyopneumoniae field isolates. Antimicrob. Agents Chemother. 48, 4470-4472.

Vicca, J., Maes, D., Stakenborg, T., Butaye, P., Minion, F., Peeters, J., De Kruif, A., Decostere, A., Haesebrouck, F., 2007. Resistance mechanism against fluoroquinolones in Mycoplasma hyopneumoniae field isolates. Microb. Drug Resist. 13, 166-170.

Waites, K.B., Duffy, L.B., Bebear, C.M., Matlow, A., Talkington, D.F., Kenny, G.E., Totten, P.A., Bade, D.J., Zheng, X., Davidson, M.K., Shortridge, V.D., Watts, J.L., Brown, D.S., 2012. Standardized methods and quality control limits for agar and broth microdilution susceptibility testing of Mycoplasma pneumoniae, Mycoplasma hominis, and Ureaplasma urealyticum. J. Clin. Microbiol. 50, 3542-3547.

Wu, C.C., Shryock, T.R., Lin, T.L., Veenhuizen, M.F., 1997. Testing antimicrobial susceptibility against Mycoplasma hyopneumoniae in vitro. Swine Health Prod. 5, $227-230$. 